\title{
Local invariants and pairwise entanglement in symmetric multiqubit system
}

\author{
A. R. Usha Devi, ${ }^{1, *}$ M. S. Uma, ${ }^{1}$ R. Prabhu,${ }^{1}$ and Sudha ${ }^{2}$ \\ 1 Department of Physics, Bangalore University, Bangalore-560 056, India \\ ${ }^{2}$ Department of Physics, Thunga First Grade College, \\ Thirthahalli-577432, India. \\ (Dated: October 11, 2018)
}

\begin{abstract}
Pairwise entanglement properties of a symmetric multi-qubit system are analyzed through a complete set of two-qubit local invariants. Collective features of entanglement, such as spin squeezing, are expressed in terms of invariants and a classification scheme for pairwise entanglement is proposed. The invariant criteria given here are shown to be related to the recently proposed (Phys. Rev. Lett. 95, 120502 (2005)) generalized spin squeezing inequalities for pairwise entanglement in symmetric multi-qubit states.

PACS numbers: 03.67.-a, 03.65.-w

Keywords: Spin squeezing; local invariants; symmetric multi-qubits; pairwise entanglement.
\end{abstract}

\section{INTRODUCTION}

Considerable interest has been evinced recently 1, 2, 3, 4, 5, 6, 7, 8, 9, 10, 11] in producing, controlling and manipulating entangled multi-atom systems due to the possibility of applications in atomic interferometry [12, 13], high precession atomic clocks [14], quantum computation and quantum information processing [15]. Multi-atom systems, which are symmetric under permutation of the particles, allow for an elegant description in terms of collective variables of the system. Specifically, if we have $N$ two level atoms, each atom may be represented as a spin- $\frac{1}{2}$ system (a qubit) and theoretical analysis in terms of collective spin operator $\vec{S}=\frac{1}{2} \sum_{\alpha=1}^{N} \vec{\sigma}_{\alpha}, \quad\left(\vec{\sigma}_{\alpha}\right.$ denote the Pauli spin operator of the $\alpha^{\text {th }}$ qubit), leads to reduction of the dimension of the Hilbert space from $2^{N}$ to $(N+1)$, when the two level multi-atom system respects exchange symmetry. A large number of experimentally relevant multi-atom states exhibit symmetry under interchange of atoms, facilitating a significant simplification in understanding the properties of the physical system.

While complete characterization of multi-particle entanglement still remains a major task, collective behaviour such as spin squeezing 1, 2, 3, 4, 5, 6, 7, 8, 9, 16, 17, 18, 19, 20, 21, 22, 23, 24, 25, 26, 27], exhibited by multi-atom systems, has been proposed as a signature of quantum correlation between the atoms. A connection between spin squeezing and the nature of quantum entanglement has been explored [26, 27] and it is shown that the presence of spin squeezing essentially reflects pairwise entanglement. However, it is important to realize that spin squeezing serves only as a sufficient condition - not a necessary one - for pairwise entanglement. There will still be pairwise correlated states, which do not exhibit spin squeezing. In a class of symmetric mul-

*Electronic address: arutth@rediffmail.com tiqubit states it has been shown 27] that spin-squeezing and pairwise entanglement imply each other. Questions like "Are there any other collective signatures of pairwise entanglement?" are still being investigated. Recently, inequalities generalizing the concept of spin squeezing have been derived [28]. These inequalities are shown to provide necessary and sufficient conditions for pairwise entanglement and three-party entanglement in symmetric $N$-qubit states. In this paper, we propose a complete characterization and classification of pairwise entanglement in symmetric multi-qubit systems, in terms of local invariants associated with a random pair of qubits drawn from the collective system. We show that pairwise entanglement in symmetric $N$-qubit states is reflected through negative values of some of the two-qubit invariants. Specifically, a symmetric multi-qubit system is spin squeezed iff one of the entanglement invariant is negative. We discuss a classification scheme for pairwise entanglement in symmetric $N$-qubit states based on non-positive values of the local invariants. We show that our characterization is indeed related to the generalized spin squeezing inequalities of Ref. 28]. In the light of our characterization, we analyze physical examples of symmetric multi-qubit states like (i) Dicke state, (ii) Kitagawa-Ueda state generated by one axis twisting Hamiltonian and (iii) Steady state of atoms irradiated by a squeezed bath.

\section{LOCAL INVARIANTS OF A SYMMETRIC TWO-QUBIT SYSTEM}

A symmetric $N$-qubit system remains unchanged by permutations of the qubits:

$$
\Pi_{\alpha \beta} \rho_{N} \Pi_{\alpha \beta}^{-1}=\rho_{N},
$$

where $\Pi_{\alpha \beta}$ denotes the permutation operator interchanging $\alpha^{\text {th }}$ and $\beta^{\text {th }}$ qubits of the multi-qubit system. Owing to this symmetry, quantum states of symmetric multiqubits get restricted to a $(2 S+1)$ dimensional subspace $\left\{\left|S=\frac{N}{2}, M\right\rangle ;-S \leq M \leq S\right\}$ of the entire $2^{N}$ dimensional Hilbert space $C^{2} \otimes C^{2} \ldots \otimes C^{2}$. Here, $|S, M\rangle$ 
are the simultaneous eigen states of the squared collective spin operator $S^{2}$ and $S_{3}$, the $z$-component of the collective operator $\vec{S}=\frac{1}{2} \sum_{\alpha=1}^{N} \vec{\sigma}_{\alpha}$.

In a symmetric $N$-qubit system the average values of collective spin correlations, upto second order, are expressed in terms of their two-qubit counterparts as follows:

$$
\left\langle S_{i}\right\rangle=\frac{1}{2} \sum_{\alpha=1}^{N}\left\langle\sigma_{\alpha i}\right\rangle=\frac{N}{2} s_{i}
$$

and

$$
\begin{aligned}
\frac{1}{2}\left\langle\left(S_{i} S_{j}+S_{j} S_{i}\right)\right\rangle & =\frac{1}{8} \sum_{\alpha, \beta=1}^{N}\left\langle\left(\sigma_{\alpha i} \sigma_{\beta j}+\sigma_{\beta i} \sigma_{\alpha j}\right)\right\rangle \\
& =\frac{1}{4} \sum_{\alpha, \beta=1}^{N}\left\langle\left(\sigma_{\alpha i} \sigma_{\beta j}\right)\right\rangle \\
& =\frac{N}{4} \delta_{i j}+\frac{N(N-1)}{4}\left\langle\left(\sigma_{1 i} \sigma_{2 j}\right)\right\rangle \\
& =\frac{N}{4}\left[\delta_{i j}+(N-1) t_{i j}\right], \quad i, j=1,2,3
\end{aligned}
$$

In the above equation, we have denoted the expectation values of the two qubit observables by 29

$$
\begin{gathered}
\left\langle\sigma_{1 i}\right\rangle=\left\langle\sigma_{2 i}\right\rangle=s_{i}, \quad \text { and } \\
\left\langle\left(\sigma_{1 i} \sigma_{2 j}\right)\right\rangle=\left\langle\left(\sigma_{1 j} \sigma_{2 i}\right)\right\rangle=t_{i j} ; \quad i, j=1,2,3 .
\end{gathered}
$$

Here, $s_{i}$ are the components of average spins of the qubits and $t_{i j}=t_{j i}$ are the elements of the $3 \times 3$ real, symmetric two-qubit correlation matrix. Pairwise entanglement properties of a symmetric multiqubit state are characterised in terms of the symmetric two-qubit density matrix given by

$$
\rho^{\mathrm{sym}}=\frac{1}{4}\left(1+\sum_{i=1}^{3}\left(\sigma_{1 i}+\sigma_{2 i}\right) s_{i}+\sum_{i, j=1}^{3}\left(\sigma_{1 i} \sigma_{2 j}\right) t_{i j}\right),
$$

which is associated with any random pair of qubits belonging to a symmetric multi-qubit ensemble.

Since the squared collective spin operator should satisfy the condition, $\sum_{i=1}^{3}\left\langle S_{i}^{2}\right\rangle=S(S+1)=\frac{N}{2}\left(\frac{N}{2}+1\right)$ we have $\frac{1}{4}\left\langle\left(\vec{\sigma}_{1}+\vec{\sigma}_{2}\right)^{2}\right\rangle=2$ at the two-qubit level, which leads to the constraint $\operatorname{Tr}(T)=1$, on the two-qubit correlation matrix $T$. Therefore, 8 state parameters - viz., three components $s_{i}$ of the average qubit orientation and the elements $t_{i j}$ of the real, symmetric correlation matrix with unit trace - characterize the two-qubit partition of the system completely.
Entanglement of a composite quantum system remain invariant, when the subsystems are subjected to local unitary operations. Any two quantum states are entanglementwise equivalent iff they are related to each other through local unitary transformations. Non-separability of a quantum state can be represented by complete set of local invariants $30,31,32,33]$ and any quantitative measure of entanglement must be a function of these invariants. In the following Theorem, we show that a set containing six local invariants provide a complete characterization of entanglement in a symmetric two-qubit system. This result proves to be useful in analysing pairwise entanglement properties of an arbitrary symmetric multi-qubit system.

Theorem 1. All equally entangled symmetric two-qubit states have identical values for the local invariants $\left\{\mathcal{I}_{1}-\right.$ $\left.\mathcal{I}_{6}\right\}$ given below:

$$
\begin{gathered}
\mathcal{I}_{1}=\operatorname{det} T, \quad \mathcal{I}_{2}=\operatorname{Tr}\left(T^{2}\right), \\
\mathcal{I}_{3}=s^{T} s, \quad \mathcal{I}_{4}=s^{T} T s, \\
\mathcal{I}_{5}=\epsilon_{i j k} \epsilon_{l m n} s_{i} s_{l} t_{j m} t_{k n}, \\
\mathcal{I}_{6}=\epsilon_{i j k} s_{i}(T s)_{j}\left(T^{2} s\right)_{k},
\end{gathered}
$$

where $\epsilon_{i j k}$ denotes Levi-Civita symbol; $s\left(s^{T}\right)$ is a column (row) with $s_{1}, s_{2}$ and $s_{3}$ as elements.

Proof: Let us first note that the state parameters of a symmetric two-qubit density matrix transform under identical local unitary operation [34] $U \otimes U$ as follows:

$$
\begin{aligned}
& s_{i}^{\prime}=\sum_{j=1}^{3} O_{i j}^{(1)} s_{j} \quad \text { or } \quad s^{\prime}=O s, \\
& t_{i j}^{\prime}=\sum_{k, l=1}^{3} O_{i k}^{(1)} O_{j l}^{(2)} t_{k l} \quad \text { or } \quad T^{\prime}=O T O^{T},
\end{aligned}
$$

where $O \in S O(3, R)$ denotes $3 \times 3$ rotation matrix, corresponding uniquely to the $2 \times 2$ unitary matrix $U \in S U(2)$.

It is easy to verify that $\left\{\mathcal{I}_{1}-\mathcal{I}_{6}\right\}$ given by Eq. (4) are all invariant, when the state variables $s$ and $T$ transform under identical local rotations as shown in Eq. (5). We may choose to specify the state parameters $s$ and $T$ in a basis in which $T$ is diagonal. This is possible because the real, symmetric correlation matrix $T$ can be diagonalized through identical local rotations:

$$
T^{d}=O T O^{T}=\operatorname{diag}\left(t_{1}, t_{2}, t_{3}\right) .
$$

It is clear that the invariants $\mathcal{I}_{1}, \mathcal{I}_{2}$, (along with the unit trace condition $\operatorname{Tr}(T)=1$ ), determine the eigenvalues $t_{1}, t_{2}$ and $t_{3}$ of the two-qubit correlation matrix $T$. Further, the absolute values of the state variables $s_{1}, s_{2}, s_{3}$, can be evaluated using $\mathcal{I}_{3}, \mathcal{I}_{4}$ and $\mathcal{I}_{5}$ :

$$
\begin{aligned}
& \mathcal{I}_{3}=s_{1}^{2}+s_{2}^{2}+s_{3}^{2}, \\
& \mathcal{I}_{4}=s_{1}^{2} t_{1}+s_{2}^{2} t_{2}+s_{3}^{2} t_{3}, \\
& \mathcal{I}_{5}=2\left(s_{1}^{2} t_{2} t_{3}+s_{2}^{2} t_{1} t_{3}+s_{3}^{2} t_{1} t_{2}\right) .
\end{aligned}
$$


The overall sign of the product $s_{1} s_{2} s_{3}$ is then fixed by $\mathcal{I}_{6}$ :

$\mathcal{I}_{6}=s_{1} s_{2} s_{3}\left[t_{1} t_{2}\left(t_{2}-t_{1}\right)+t_{2} t_{3}\left(t_{3}-t_{2}\right)+t_{3} t_{1}\left(t_{1}-t_{3}\right)\right]$.

It is important to realize that only the overall sign of $s_{1} s_{2} s_{3}$ - not the individual signs - is a local invariant. More explicitly, if $(+,+,+)$ denote the signs of $s_{1}, s_{2}$ and $s_{3}$, identical local rotation through an angle $\pi$ about the axes $x, y$ or $z$ affect only the signs, not the magnitudes of $s_{1}, s_{2}, s_{3}$, leading to the possibilities $(+,-,-),(-,+,-),(-,-,+)$. All these combinations correspond to the ' + ' sign for the product $s_{1} s_{2} s_{3}$. Similarly, the overall '-' sign for the product $s_{1} s_{2} s_{3}$ arises from the combinations, $(-,-,-),(-,+,+),(+,-,+)$, $(+,+,-)$, which are all related to each other by $180^{\circ}$ local rotations about the $x, y$ or $z$ axes.

Thus we have shown that every symmetric two qubit density matrix can be transformed by identical local unitary transformation $U \otimes U$ to a canonical form, specified completely by the set of invariants $\left\{\mathcal{I}_{1}-\mathcal{I}_{6}\right\}$. In other words, symmetric two-qubit states are equally entangled iff $\left\{\mathcal{I}_{1}-\mathcal{I}_{6}\right\}$ are same. $\square$

Now, we proceed to identify criteria of pairwise entanglement in a symmetric multi-qubit state, in terms of the two-qubit local invariants $\left\{\mathcal{I}_{1}-\mathcal{I}_{6}\right\}$.

We first note that the invariants $\mathcal{I}_{2}, \mathcal{I}_{3} \geq 0$ for all symmetric states. The signs of other invariants play a significant role in characterizing entanglement.

Theorem 2. The invariants $\mathcal{I}_{1}, \mathcal{I}_{4}, \mathcal{I}_{5}$ and the combination $\mathcal{I}_{4}-\mathcal{I}_{3}^{2}$ are non-negative in a symmetric separable state.

Proof: A separable symmetric multi-qubit state is given by

$$
\rho_{(\mathrm{sym}-\mathrm{sep})}=\sum_{w} p_{w} \rho^{(w)} \otimes \rho^{(w)} \otimes \ldots \rho^{(w)}
$$

with $\sum_{w} p_{w}=1$ and $\rho^{(w)}=\frac{1}{2}\left(1+\sum_{i=1}^{3} \sigma_{i} s_{i}^{w}\right)$ denote single qubit density matrix. Two qubit partition of the separable symmetric system is given by

$$
\begin{aligned}
\rho_{(\mathrm{sym}-\mathrm{sep})}^{(\alpha \beta)} & =\operatorname{Tr}_{\{1,2, \ldots, N / \alpha, \beta\}}\left(\rho_{(\mathrm{sym}-\mathrm{sep})}\right) \\
& =\sum_{w} p_{w} \rho^{(w)} \otimes \rho^{(w)}
\end{aligned}
$$

In Eq. (10), the two-qubit density matrix $\rho_{(\mathrm{sym}-\mathrm{sep})}^{(\alpha \beta)}$ is independent of the qubit indices $\alpha, \beta$, owing to exchange symmetry. The state variables of the two-qubit separable symmetric state are given by

$$
\begin{aligned}
s_{i} & =\operatorname{Tr}\left(\rho_{(\mathrm{sym}-\mathrm{sep})}^{(\alpha \beta)} \sigma_{i}\right)=\sum_{w} p_{w} s_{i}^{(w)} \\
t_{i j} & =\operatorname{Tr}\left(\rho_{(\mathrm{sym}-\mathrm{sep})}^{(\alpha \beta)} \sigma_{\alpha i} \sigma_{\beta j}\right)=\sum_{w} p_{w} s_{i}^{(w)} s_{j}^{(w)}
\end{aligned}
$$

(i) To prove that $\mathcal{I}_{1} \geq 0$ in a separable symmetric state, we transform $\rho_{(\mathrm{sym}-\mathrm{sep})}^{(\alpha \beta)}$ with the help of a suitable local rotation such that $T=\operatorname{diag}\left(t_{1}, t_{2}, t_{3}\right)=$ $\operatorname{diag}\left(\sum_{w} p_{w}\left(s_{1}^{(w)}\right)^{2}, \sum_{w} p_{w}\left(s_{2}^{(w)}\right)^{2}, \sum_{w} p_{w}\left(s_{3}^{(w)}\right)^{2}\right)$.

We therefore have

$$
\mathcal{I}_{1}=\operatorname{det} T=t_{1} t_{2} t_{3}=\prod_{i=1}^{3}\left(\sum_{w} p_{w}\left(s_{i}^{(w)}\right)^{2}\right),
$$

which is obviously non-negative.

(ii) The invariant $\mathcal{I}_{4}$ has the following structure for a separable state:

$$
\begin{aligned}
\mathcal{I}_{4} & =s^{T} T s=\sum_{i, j=1}^{3} t_{i j} s_{i} s_{j} \\
& =\sum_{w} p_{w}\left(\sum_{i=1}^{3} s_{i}^{(w)} s_{i}\right)\left(\sum_{j=1}^{3} s_{j}^{(w)} s_{j}\right) \\
& =\sum_{w} p_{w}\left(\vec{s} \cdot \vec{s}^{(w)}\right)^{2} \geq 0 .
\end{aligned}
$$

(iii) Now, consider the invariant $\mathcal{I}_{5}$ for a separable symmetric system:

$$
\begin{aligned}
\mathcal{I}_{5} & =\epsilon_{i j k} \epsilon_{l m n} s_{i} s_{l} t_{j m} t_{k n} \\
& =\sum_{w, w^{\prime}} p_{w} p_{w^{\prime}}\left(\epsilon_{i j k} s_{i} s_{j}^{(w)} s_{k}^{\left(w^{\prime}\right)}\right)\left(\epsilon_{l m n} s_{l} s_{m}^{(w)} s_{n}^{\left(w^{\prime}\right)}\right) \\
& =\sum_{w, w^{\prime}} p_{w} p_{w^{\prime}}\left[\vec{s} \cdot\left(\vec{s}^{(w)} \times \vec{s}^{\left(w^{\prime}\right)}\right)\right]^{2} \geq 0 .
\end{aligned}
$$

(iv) For the combination $\mathcal{I}_{4}-\mathcal{I}_{3}^{2}$ we obtain,

$$
\mathcal{I}_{4}-\mathcal{I}_{3}^{2}=\sum_{w} p_{w}\left(\vec{s} \cdot \vec{s}^{(w)}\right)^{2}-\left(\sum_{w} p_{w}\left(\vec{s} \cdot \vec{s}^{(w)}\right)\right)^{2}
$$

which has the structure $\left\langle A^{2}\right\rangle-\langle A\rangle^{2}$ and is therefore, essentially non-negative.

Negative value assumed by any of the invariants $\mathcal{I}_{1}, \mathcal{I}_{4}, \mathcal{I}_{5}$ or the $\mathcal{I}_{3}-\mathcal{I}_{4}^{2}$, is a signature of pairwise entanglement. Moreover, from the structure of the invariants in a symmetric separable state, it is clear that $\mathcal{I}_{4}=\sum_{w} p_{w}\left(\vec{s} \cdot \vec{s}^{(w)}\right)^{2}=0$ implies $\vec{s}^{(w)} \equiv 0$ for all ' $w$ ', leading in turn to $\mathcal{I}_{3}=\sum_{w} p_{w}\left(\vec{s} \cdot \vec{s}^{(w)}\right)=0$ and $\mathcal{I}_{5}=0$ (see Eq. (15)). So, $\mathcal{I}_{3} \neq 0, \mathcal{I}_{4}, \mathcal{I}_{5} \leq 0$, reflects pairwise entanglement in symmetric multi-qubit states.

In the next section, we express collective properties, which exhibit pairwise entanglement, in terms of twoqubit invariants.

\section{COLLECTIVE SIGNATURES OF PAIRWISE ENTANGLEMENT}

Collective phenomena, reflecting pairwise entanglement of qubits, should be expressible through local in- 
variants. We begin with spin squeezing in $N$-qubit symmetric states. Spin squeezing is a manifestation of quantum correlations, resulting in the reduced quantum fluctuations in one of the collective spin components, normal to the mean spin direction, below the standard quantum limit $N / 4$ of spin coherent states [16]. A quantitative measure of this feature is given by the spin squeezing parameter,

$$
\xi^{2}=\frac{4}{N}\left(\Delta S_{\perp}\right)_{\min }^{2}
$$

where $S_{\perp}=\vec{S} \cdot \hat{n}_{\perp}$ is a perpendicular component of collective spin; $\hat{n}_{\perp}$ denotes a unit vector in the plane orthogonal to the mean spin direction $\langle\vec{S}\rangle$, and is chosen so that the variance $\left(\Delta S_{\perp}\right)^{2}$ is minimized. Symmetric multiqubit states with $\xi^{2}<1$ are spin squeezed. We now show that

Theorem 3. For all spin squeezed states, the local invariant $\mathcal{I}_{5}$ is negative.

Proof: It is useful to evaluate the invariant $\mathcal{I}_{5}$ (see Eq. (4) ), after subjecting the quantum state to a identical local rotation, which is designed to align the average spin vector $\langle\vec{S}\rangle$ along the $z$-axis i.e., for the two-qubit partition of the system, the state variable $\vec{s} \equiv\left(0,0, s_{0}\right)$ after this local operation. We now obtain,

$$
\begin{aligned}
\mathcal{I}_{5} & =\epsilon_{3 j k} \epsilon_{3 m n} s_{0}^{2} t_{j m} t_{k n} \\
& =2 s_{0}^{2}\left(t_{11} t_{22}-t_{12}^{2}\right) \\
& =2 s_{0}^{2} \operatorname{det} T_{\perp},
\end{aligned}
$$

where $T_{\perp}$ denotes the $2 \times 2$ block of the correlation matrix in the subspace orthogonal to the qubit orientation direction i.e., z-axis. Now, we can still exploit the freedom of local rotations in the $x-y$ plane, which leaves the average spin $\vec{s}=\left(0,0, s_{0}\right)$ unaffected. We use this to diagonalize $T_{\perp}$ :

$$
T_{\perp}=\left(\begin{array}{ll}
t_{\perp}^{(+)} & 0 \\
0 & t_{\perp}^{(-)}
\end{array}\right)
$$

with $t_{\perp}^{( \pm)}=\frac{1}{2}\left[\left(t_{11}+t_{22}\right) \pm \sqrt{\left(t_{11}-t_{22}^{2}\right)+4 t_{12}^{2}}\right]$. We thus obtain,

$$
\mathcal{I}_{5}=2 s_{0}^{2} t_{\perp}^{(+)} t_{\perp}^{(-)}
$$

We now express the spin squeezing parameter $\xi^{2}$, given by Eq. (17), in terms of the two-qubit state parameters:

$$
\begin{aligned}
\xi^{2} & =\frac{4}{N}\left\langle\left(\vec{S} \cdot \hat{n}_{\perp}\right)^{2}\right\rangle_{\min } \\
& =\frac{1}{N} \sum_{\alpha, \beta=1}^{N}\left\langle\left(\vec{\sigma}_{\alpha} \cdot \hat{n}_{\perp}\right)\left(\vec{\sigma}_{\beta} \cdot \hat{n}_{\perp}\right)\right\rangle_{\min } \\
& =1+\frac{1}{N} \sum_{\alpha=1}^{N} \sum_{\beta \neq \alpha=1}^{N}\left\langle\left(\vec{\sigma}_{\alpha} \cdot \hat{n}_{\perp}\right)\left(\vec{\sigma}_{\beta} \cdot \hat{n}_{\perp}\right)\right\rangle_{\min } \\
& =1+\frac{2}{N} \sum_{\alpha=1}^{N} \sum_{\beta>\alpha=1}^{N}\left(\sum_{i, j=1}^{3}\left\langle\left(\sigma_{\alpha i} \sigma_{\beta} j\right)\right\rangle n_{\perp i} n_{\perp j}\right)_{\min } .
\end{aligned}
$$

For a symmetric system, $\left\langle\sigma_{\alpha i} \sigma_{\beta j}\right\rangle=t_{i j}$, - independent of the qubit indices $\alpha, \beta$ - and we obtain,

$$
\begin{aligned}
\xi^{2} & =1+(N-1)\left(\sum_{i, j=1}^{3} t_{i j} n_{\perp i} n_{\perp j}\right)_{\min } \\
& =1+(N-1)\left(n_{\perp}^{T} T n_{\perp}\right)_{\min } .
\end{aligned}
$$

In Eq. (22), we have denoted the row vector $n_{\perp}^{T}=\left(n_{1 \perp}, n_{2 \perp}, n_{3 \perp}\right)$.

With the mean spin along the $z$ direction, we have $\hat{n}_{\perp}=(\cos \theta, \sin \theta, 0)$, and the minimum value of the quadratic form $\left(n_{\perp}^{T} T n_{\perp}\right)_{\min }$ in Eq. (22) is fixed as follows:

$$
\begin{aligned}
\left(n_{\perp}^{T} T n_{\perp}\right)_{\min } & =\min _{\theta}\left(t_{11} \cos ^{2} \theta+t_{22} \sin ^{2} \theta+t_{12} \sin 2 \theta\right) \\
& =\frac{1}{2}\left[\left(t_{11}+t_{22}\right)-\sqrt{\left(t_{11}-t_{22}^{2}\right)+4 t_{12}^{2}}\right] \\
& =t_{\perp}^{(-)},
\end{aligned}
$$

where $t_{\perp}^{(-)}$is the least eigenvalue of $T_{\perp}$ (see Eq. (19)). We finally have

$$
\xi^{2}=\frac{4}{N}\left(\Delta S_{\perp}\right)_{\min }^{2}=\left(1+(N-1) t_{\perp}^{(-)}\right) .
$$

Following similar lines we can also show that

$$
\frac{4}{N}\left(\Delta S_{\perp}\right)_{\max }^{2}=\left(1+(N-1) t_{\perp}^{(+)}\right),
$$

which relates the eigenvalue $t_{\perp}^{(+)}$of $T_{\perp}$ to the maximum collective fluctuation $\left(\Delta S_{\perp}\right)_{\max }^{2}$ orthogonal to the mean spin direction. Substituting Eqs. (24), (25) and expressing $s_{0}=\frac{2}{N}|\langle\vec{S}\rangle|$ in Eq. (20), we get

$$
\mathcal{I}_{5}=\frac{8|\langle\vec{S}\rangle|^{2}}{(N(N-1))^{2}}\left(\xi^{2}-1\right)\left(\frac{4}{N}\left(\Delta S_{\perp}\right)_{\max }^{2}-1\right) .
$$

Having related the local invariant $\mathcal{I}_{5}$ to collective spin observables, we now proceed to show that $\mathcal{I}_{5}<0$ iff $\xi^{2}<1$ i.e., iff the state is spin squeezed. 
It has been shown 35] that positivity of any arbitrary two-qubit density operator imposes the bound $-1 \leq t_{11}, t_{22}, t_{33} \leq 1$, on the diagonal elements of the correlation matrix $T$. This bound, together with the unit trace condition $\operatorname{Tr}(T)=1$ on the correlation matrix of a symmetric two-qubit state, leads to the identification that only one of the diagonal elements of $T$ can be negative. This in turn implies that $t_{\perp}^{(+)}=\frac{1}{N-1}\left(\frac{4}{N}\left(\Delta S_{\perp}\right)_{\max }^{2}-1\right) \geq 0$.

Therefore, a symmetric multiqubit state is spinsqueezed $\left(\xi^{2}<1\right)$ iff the local invariant $\mathcal{I}_{5}<0$. $\square$

Further, from the structure of the invariant $\mathcal{I}_{5}$ given in Eq. (7), it is clear that one of the eigenvalues $t_{1}, t_{2}$ or $t_{3}$ of the correlation matrix $T$ must be negative in order that $\mathcal{I}_{5}<0$. Thus the invariant $\mathcal{I}_{1}=t_{1} t_{2} t_{3}$ is also negative, when the system is spin squeezed.

We now explore other collective signatures of pairwise entanglement, which are manifestations of negative values of the invariants $\mathcal{I}_{4}$ and $\mathcal{I}_{4}-\mathcal{I}_{3}^{2}$.

It is easy to see that the invariant $\mathcal{I}_{4}$ assumes a simple form, when the average spin vector is oriented along the $z$-axis:

$$
\mathcal{I}_{4}=s^{T} T s=s_{0}^{2} t_{33}
$$

So, $\mathcal{I}_{4}$ is negative iff $t_{33}<0$. Using Eqs. (1) and (2), we express $\mathcal{I}_{4}$ in terms of collective observables as

$$
\mathcal{I}_{4}=\frac{4}{N^{2}(N-1)}|\langle\vec{S}\rangle|^{2}\left(\frac{4}{N}\left\langle(\vec{S} \cdot \hat{n})^{2}\right\rangle-1\right),
$$

where $\hat{n}$ denotes a unit vector along the direction of mean spin. We therefore read from Eq. (28), that the average of the squared spin component, along the mean spin direction, reduced below the value $N / 4$, signifies pairwise entanglement in symmetric $N$-qubit system.

We note that $\mathcal{I}_{5}$ is not negative, when $\mathcal{I}_{4} \leq 0$. This is because $t_{33} \leq 0$ implies $t_{\perp}^{( \pm)} \geq 0$, as $t_{\perp}^{(+)}+t_{\perp}^{(-)}+t_{33}=1$, (unit trace condition) and $-1 \leq t_{\perp}^{( \pm)}, t_{33} \leq 1$, (positivity condition [35]). Therefore, spin squeezing and $\left\langle(\vec{S} \cdot \hat{n})^{2}\right\rangle \leq \frac{N}{4}$ are two mutually exclusive criteria of pairwise entanglement. However, it is obvious from the structure of the invariant $\mathcal{I}_{4}$, as given in Eq. (7), that $\mathcal{I}_{4} \leq 0$ implies $\mathcal{I}_{1}=t_{1} t_{2} t_{3} \leq 0$.

Now we relate $\mathcal{I}_{4}-\mathcal{I}_{3}^{2}$ to collective variables:

$$
\begin{aligned}
\mathcal{I}_{4}-\mathcal{I}_{3}^{2} & =s_{0}^{2}\left(t_{33}-s_{0}^{2}\right) \\
& =\frac{4}{N^{2}}|\langle\vec{S}\rangle|^{2}\left(\frac{4}{N(N-1)}\left\langle(\vec{S} \cdot \hat{n})^{2}\right\rangle-\frac{1}{(N-1)}-\frac{4}{N^{2}}|\langle\vec{S}\rangle|^{2}\right) \\
& =\frac{16}{N^{3}(N-1)}|\langle\vec{S}\rangle|^{2}\left(\left\langle(\vec{S} \cdot \hat{n})^{2}\right\rangle-\frac{N}{4}-\frac{(N-1)}{N}|\langle\vec{S}\rangle|^{2}\right) .
\end{aligned}
$$

Negative value of the combination $\mathcal{I}_{4}-\mathcal{I}_{3}^{2}$ manifests itself through $\left\langle(\vec{S} \cdot \hat{n})^{2}\right\rangle<\frac{N}{4}+\frac{(N-1)}{N}|\langle\vec{S}\rangle|^{2}$. From Eqs. (28) and (29), we conclude that pairwise entanglement resulting from $\mathcal{I}_{3} \neq 0, \mathcal{I}_{4}>0$, but $\mathcal{I}_{4}-\mathcal{I}_{3}^{2}<0$, is realised, whenever

$$
\frac{N}{4}<\left\langle(\vec{S} \cdot \hat{n})^{2}\right\rangle<\frac{N}{4}+\frac{(N-1)}{N}|\langle\vec{S}\rangle|^{2} .
$$

In the cases, where the qubits have no preferred orientation, i.e., when $|\langle\vec{S}\rangle|=0$, all the local invariants except $\mathcal{I}_{1}$ and $\mathcal{I}_{2}$ are zero. In such situations, pairwise entanglement manifests itself [36] $\mathcal{I}_{1}<0$. In terms of collective observables, we have

$$
\mathcal{I}_{1}=\left(\frac{4}{N(N-1)}\right)^{3} \prod_{i=1}^{3}\left(\left\langle S_{i}^{2}\right\rangle-\frac{N}{4}\right) .
$$

Negative value of $\mathcal{I}_{1}$ shows up through $\left\langle S_{i}^{2}\right\rangle<\frac{N}{4}$ along the axes $i=1,2$ or 3 , which are fixed by verifying $\left\langle\left(S_{i} S_{j}+S_{j} S_{i}\right)\right\rangle=0 ; i \neq j$, as $T$ is diagonal with such a choice of the axes.
Finally, we arrive at a classification scheme, as depicted in Table.1, for pairwise entanglement in symmetric multiqubit states. Next, we proceed to relate our invariant criteria with the recently proposed generalized spin squeezing inequalities [28] for pairwise entanglement

$$
\frac{4\left\langle\Delta S_{k}\right\rangle^{2}}{N}<1-\frac{4\left\langle S_{k}^{2}\right\rangle}{N^{2}}
$$

where $S_{k}=\vec{S} \cdot \hat{k}$; with $\hat{k}$ denoting an arbitrary unit vector. We consider different situations as discussed below: (i) Let us now choose $\hat{k}=\hat{n}_{\perp}$, a direction orthogonal to the mean spin vector $\langle\vec{S}\rangle$. The inequality given by Eq. (31) reduces to

$$
\left(\Delta S_{n_{\perp}}\right)^{2}<\frac{N}{4}
$$

Minimizing the left hand side of this inequality gives $\left(\Delta S_{\perp}\right)_{\min }^{2}<\frac{N}{4}$ and corresponds to the condition $\mathcal{I}_{5}<0$ on the local invariant.

(ii) If $\hat{k}$ is aligned along the mean spin direction i.e., $\hat{k}=\hat{n}$ 
TABLE I: Classification of pairwise entanglement in terms of two-qubit local invariants.

\begin{tabular}{|c|c|c|}
\hline \multicolumn{1}{|c|}{ Criterion of pairwise entanglement } & Collective behaviour to look for \\
\hline \multirow{5}{*}{$\mathcal{I}_{3} \neq 0$} & $\mathcal{I}_{5} \leq 0$ & $\left(\Delta S_{\perp}\right)_{\min }^{2} \leq \frac{N}{4}$ \\
\cline { 2 - 3 } & $\mathcal{I}_{4} \leq 0$ & $\left\langle(\vec{S} \cdot \hat{n})^{2}\right\rangle \leq \frac{N}{4}$ \\
\cline { 2 - 3 } & $\mathcal{I}_{4}>0, \mathcal{I}_{4}-\mathcal{I}_{3}^{2}<0$ & $\frac{N}{4}<\left\langle(\vec{S} \cdot \hat{n})^{2}\right\rangle<\frac{N}{4}+\frac{(N-1)}{N}|\langle\vec{S}\rangle|^{2}$ \\
\hline \multirow{2}{*}{$\mathcal{I}_{3}=0$} & $\mathcal{I}_{1}<0$ & $\begin{array}{c}\left\langle S_{i}^{2}\right\rangle<\frac{N}{4} \\
\text { for any direction } i=1,2,3, \text { so that } \\
\left\langle\left(S_{i} S_{j}+S_{j} S_{i}\right)\right\rangle=0 ; \text { for } i \neq j\end{array}$ \\
\hline
\end{tabular}

with $\hat{n}=\frac{\langle\vec{S}\rangle}{|\langle\vec{S}\rangle|}$, the generalized spin squeezing inequalities (see Eq. (31) ) reduce to the form,

$$
\left\langle(\vec{S} \cdot \hat{n})^{2}\right\rangle<\frac{N}{4}+\frac{(N-1)}{N}|\langle\vec{S}\rangle|^{2} .
$$

Note that the condition $\mathcal{I}_{4}<0$ on the local invariant leads to the collective signature (see Table.1)

$$
\left\langle(\vec{S} \cdot \hat{n})^{2}\right\rangle<\frac{N}{4}
$$

which is a stronger restriction than that given by Eq. (32). Further, if $\mathcal{I}_{4}<0$ but $\mathcal{I}_{4}-\mathcal{I}_{3}^{2}<0$ we obtain the inequality (see Table.1)

$$
\frac{N}{4}<\left\langle(\vec{S} \cdot \hat{n})^{2}\right\rangle<\frac{N}{4}+\frac{(N-1)}{N}|\langle\vec{S}\rangle|^{2}
$$

which covers the remaining range of possibilties contained in the generalized spin squeezing inequalities of Eq. (32) with $\hat{n}$ along the mean spin direction.

(iii) If the average spin is zero for a given state, we have $\left\langle S_{k}\right\rangle=0$ for all directions $\hat{k}$. The inequalities of Korbicz et al. 28] assume a simple form $\left\langle S_{k}\right\rangle^{2}<\frac{N}{4}$ in this case, which obviously corresponds to $\mathcal{I}_{3}=0$ and $\mathcal{I}_{1}<0$.

Now we consider specific examples of symmetric multiqubit states.

\section{A. Dicke state}

Dicke states, $\left|S=\frac{N}{2}, M\right\rangle ;-S \leq M \leq S$, are collective symmetric multi-atom states, which were shown [37] to exhibit enhanced spontaneous emission rates (superradiance) in atom-field interactions. For any random pair of atoms, drawn from a Dicke state, the state variables are given by

$$
\begin{aligned}
\vec{s} & \equiv\left(0,0, \frac{2 M}{N}\right) \\
T & =\operatorname{diag}\left(\frac{N^{2}-4 M^{2}}{2 N(N-1)}, \frac{N^{2}-4 M^{2}}{2 N(N-1)}, \frac{4 M^{2}-N}{N(N-1)}\right)
\end{aligned}
$$

The two-qubit local invariants associated with a Dicke state are

$$
\begin{aligned}
& \mathcal{I}_{1}=\left(\frac{N^{2}-4 M^{2}}{2 N(N-1)}\right)^{2}\left(\frac{4 M^{2}-N}{N(N-1)}\right), \\
& \mathcal{I}_{2}=2\left(\frac{N^{2}-4 M^{2}}{2 N(N-1)}\right)^{2}+\left(\frac{4 M^{2}-N}{N(N-1)}\right)^{2} \\
& \mathcal{I}_{3}=\frac{4 M^{2}}{N^{2}}, \quad \mathcal{I}_{4}=\mathcal{I}_{3}\left(\frac{4 M^{2}-N}{N(N-1)}\right) \\
& \mathcal{I}_{5}=8 \mathcal{I}_{3}\left(\frac{N^{2}-4 M^{2}}{4 N(N-1)}\right)^{2}, \quad \mathcal{I}_{6}=0 .
\end{aligned}
$$

Further, the combination $\mathcal{I}_{4}-\mathcal{I}_{3}^{2}$ of invariants, is given by

$$
\mathcal{I}_{4}-\mathcal{I}_{3}^{2}=\left(\frac{4 M^{2}-N^{2}}{N^{2}(N-1)}\right) \mathcal{I}_{3}
$$

We identify three different cases here: $(i) M=$ $\pm \frac{N}{2}$, (ii) $M=0$, (iii) $M \neq \pm \frac{N}{2}, 0$ Case (i) corresponds to the situation in which all the qubits are spin-up (spindown). The collective state, corresponding to this case, is obviously a product state. The invariants in this case are given by

$$
\mathcal{I}_{1}=\mathcal{I}_{5}=0, \quad \mathcal{I}_{2}=\mathcal{I}_{3}=\mathcal{I}_{4}=1,
$$

reflecting that the system is not entangled. In case (ii) the invariants $\mathcal{I}_{3}=\mathcal{I}_{4}=\mathcal{I}_{5}=0$, while the non-zero invariant

$$
\mathcal{I}_{1}=-\frac{1}{4}\left(\frac{N}{N-1}\right)^{3}
$$


assumes negative value. So, the Dicke state $\left|\frac{N}{2}, 0\right\rangle$, (with even number of atoms), exhibits pairwise entanglement. In case (iii) the invariant $\mathcal{I}_{4}$ is bound by $-\frac{1}{N-1}<\mathcal{I}_{4}<1$, and the combination $\mathcal{I}_{4}-\mathcal{I}_{3}^{2}$ is always negative, thus revealing pairwise entanglement in Dicke atoms in this case too.

\section{B. Kitagawa-Ueda state generated by one axis twisting Hamiltonian}

Kitagawa and Ueda [16 had proposed the generation of correlated $N$-qubit states, which are spin squeezed, through the Hamiltonian evolution

$$
\left|\Psi_{\mathrm{K}-\mathrm{U}}\right\rangle=e^{-i S_{3}^{2} \chi t}|S,-S\rangle ; \quad S=\frac{N}{2},
$$

referred to as one-axis twisting mechanism. The effective Hamiltonian $H=S_{3}^{2} \chi$, has already been employed to produce entangled states of four particles [38]. Collisional interactions between atoms in two-component Bose-Einstein condensation are also modeled using this one-axis twisting Hamiltonian [9].

A random pair of qubits drawn from the KitagawaUeda state are characterized by the following parameters:

$$
\vec{s}=\left(0,0,-\cos ^{(N-1)}(\chi t)\right),
$$

and the correlation matrix elements given by

$$
\begin{aligned}
t_{11} & =t_{13}=t_{23}=0, \quad t_{12}=\cos ^{(N-2)}(\chi t) \sin (\chi t), \\
t_{22} & =\frac{1}{2}\left(1-\cos ^{(N-2)}(2 \chi t)\right), \\
t_{33} & =\frac{1}{2}\left(1+\cos ^{(N-2)}(2 \chi t)\right) .
\end{aligned}
$$

We give below, the two-qubit invariants associated with the Kitagawa-Ueda state:

$\mathcal{I}_{1}=-\frac{1}{2} \cos ^{2(N-2)}(\chi t) \sin ^{2}(\chi t)\left(1+\cos ^{(N-2)}(2 \chi t)\right)$,
$\mathcal{I}_{2}=2 \cos ^{2(N-2)}(\chi t) \sin ^{2}(\chi t)+\frac{1}{2}\left(1+\cos ^{2(N-2)}(\chi t)\right)$,
$\mathcal{I}_{3}=\cos ^{2(N-1)}(\chi t), \quad \mathcal{I}_{4}=\frac{1}{2} \mathcal{I}_{3}\left(1+\cos ^{(N-2)}(2 \chi t)\right)$,
$\mathcal{I}_{5}=-2 \mathcal{I}_{3} \cos ^{2(N-2)}(\chi t) \sin ^{2}(\chi t), \quad \mathcal{I}_{6}=0$.

We see that pairwise entanglement is manifest through $\mathcal{I}_{5}<0$, collective signature of which is spin squeezing. In Fig. 1, we have plotted $\mathcal{I}_{4}, \mathcal{I}_{4}-\mathcal{I}_{3}^{2}$ and $\mathcal{I}_{5}$, for different values of $N$.

\section{Steady state of atoms irradiated by a squeezed bath}

Steady state, realized in the interaction of a collection of $N$ two-level atoms with squeezed radiation, has been
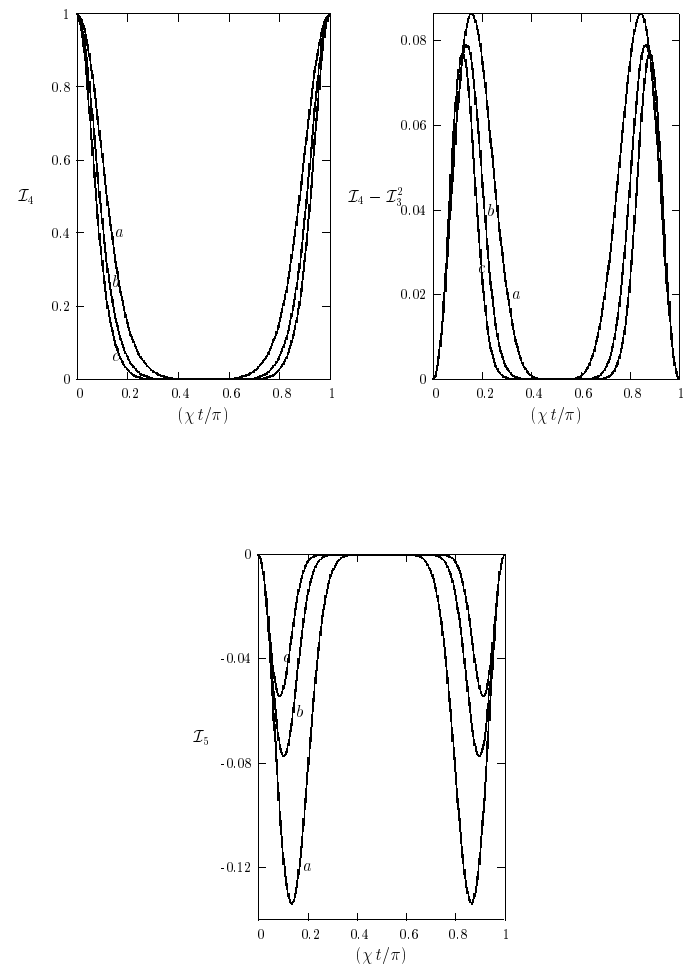

FIG. 1: The invariants $\mathcal{I}_{4}, \mathcal{I}_{4}-\mathcal{I}_{3}^{2}$ and $\mathcal{I}_{5}$, corresponding to a $N$-qubit Kitegawa-Ueda state. Curve $a: N=4, b: N=$ 6 , and $c: N=8$.

shown [17] to be a pure state (for even $N$ ) given by

$$
\left|\Psi_{0}\right\rangle=A_{0} \exp \left(\theta S_{3}\right) \exp \left(-i \frac{\pi}{2} S_{2}\right)\left|S=\frac{N}{2}, 0\right\rangle,
$$

for certain values of external field strength and detuning parameters. In Eq. (42), $\theta=\frac{1}{2} \ln (\tanh (2|\xi|)) ; \xi$ is the squeezing parameter associated with the input radiation, with mean photon number $\bar{n}=\sinh ^{2}|\xi|$ and $A_{0}$ is the normalization constant.

Two-qubit state variables, corresponding to the collective atomic system, are given by

$$
\begin{aligned}
\vec{s} & =\left(0,0, \frac{2\left\langle S_{3}\right\rangle}{N}\right), \quad T=\operatorname{diag}\left(t_{1}, t_{2}, t_{3}\right), \\
t_{1} & =\frac{-2\left\langle S_{3}\right\rangle e^{-2|\xi|}-N}{N(N-1)}, \quad t_{2}=\frac{-2\left\langle S_{3}\right\rangle e^{2|\xi|}-N}{N(N-1)} \\
t_{3} & =\frac{4\left\langle S_{3}\right\rangle \cosh (2|\xi|)+N^{2}+N}{N(N-1)},
\end{aligned}
$$



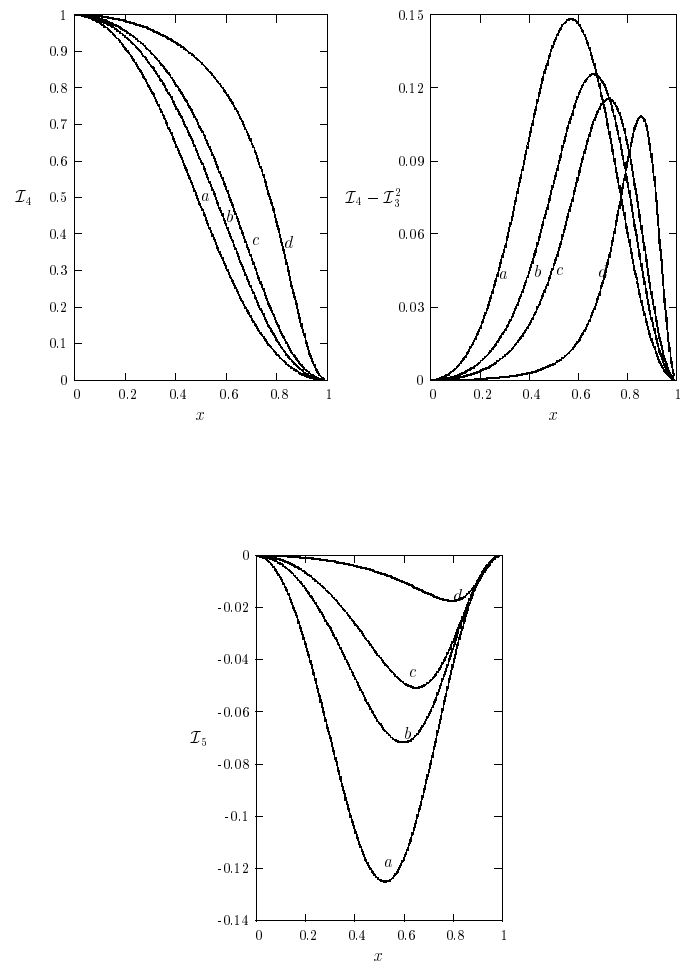

FIG. 2: The invariants $\mathcal{I}_{4}, \mathcal{I}_{4}-\mathcal{I}_{3}^{2}$ and $\mathcal{I}_{5}$, associated with the steady state of $N$ two level atoms interacting with squeezed radiation. Curve $a: N=4, b: N=6, c: N=8$, and $d$ : $N=20$.

with

$$
\left\langle S_{3}\right\rangle=\frac{\sum_{M=-S}^{S} M e^{2 M \theta}\left(d_{M 0}^{S}\left(\frac{\pi}{2}\right)\right)^{2}}{\sum_{M=-S}^{S} e^{2 M \theta}\left(d_{M 0}^{S}\left(\frac{\pi}{2}\right)\right)^{2}}
$$

and the coefficient $d_{M 0}^{S}\left(\frac{\pi}{2}\right)$ is given by [39]

$$
\begin{aligned}
& d_{M 0}^{S}\left(\frac{\pi}{2}\right)= \frac{S ! \sqrt{(S+M) !(S-M) !}}{2^{S}} \\
& \sum_{p=M}^{S-M} \frac{(-1)^{p}}{(S-M) ! p !(p-M) !(S+M-p) !} .
\end{aligned}
$$

The corresponding local invariants are listed below:

$$
\begin{aligned}
& \mathcal{I}_{1}=t_{1} t_{2} t_{3}, \quad \mathcal{I}_{2}=t_{1}^{2}+t_{2}^{2}+t_{3}^{2} \\
& \mathcal{I}_{3}=\frac{4\left\langle S_{3}\right\rangle^{2}}{N^{2}}, \quad \mathcal{I}_{4}=\mathcal{I}_{3} \frac{4\left\langle S_{3}\right\rangle \cosh (2|\xi|)+N^{2}+N}{N(N-1)} \\
& \mathcal{I}_{5}=\frac{2 \mathcal{I}_{3}}{N^{2}(N-1)^{2}}\left(2\left\langle S_{3}\right\rangle e^{-2|\xi|}+N\right)\left(2\left\langle S_{3}\right\rangle e^{2|\xi|}+N\right), \\
& \mathcal{I}_{6}=0 .
\end{aligned}
$$

In Fig. 2 we have plotted the invariants $\mathcal{I}_{4}, \mathcal{I}_{4}-\mathcal{I}_{3}^{2}$ and $\mathcal{I}_{5}$, as a function of the parameter $x=e^{2 \theta}=\sqrt{\frac{\bar{n}}{\bar{n}+1}}$, for different values of $N$. These plots demonstrate that the invariant $\mathcal{I}_{5}$ is negative, highlighting the pairwise entanglement (spin squeezing) of the atomic state.

\section{SUMMARY}

In summary, we have shown that a set of six local invariants $\left\{\mathcal{I}_{1}-\mathcal{I}_{6}\right\}$, associated with the two-qubit partition of a symmetric multiqubit system, completely characterizes the pairwise entanglement properties of the collective state. For symmetric separable states, we have proved that the entanglement invariants $\mathcal{I}_{1}, \mathcal{I}_{4}, \mathcal{I}_{5}$ and $\mathcal{I}_{4}-\mathcal{I}_{3}^{2}$ assume non-negative values. We have proposed a detailed classification scheme, for pairwise entanglement in symmetric multiqubit system, based on negative values of the invariants $\mathcal{I}_{1}, \mathcal{I}_{4}, \mathcal{I}_{5}$ and $\mathcal{I}_{4}-\mathcal{I}_{3}^{2}$. Our scheme also relates appropriate collective non-classical features, which can be identified in each case of pairwise entanglement. Specifically, we have shown, collective spin squeezing in symmetric multi-qubit states is a manifestation of $\mathcal{I}_{5}<0$. Moreover, we have related our criteria, which are essentially given in terms of invariants of the quantum state, to the recently proposed generalized spin squeezing inequalities for bipartite entanglement. We have studied (i) Dicke state, (ii) Kitagawa-Ueda state generated by the the application of one-axis twisting Hamiltonian, and (iii) the steady state of a collection of two-level atoms interacting with squeezed radiation, making use of the proposed characterization. We hope that our analysis may provide useful insights in understanding higher order quantum correlations in multi-particle states.

[1] D. J. Wineland, J. J. Bollinger, W. M. Itano, F. L. Moore, and D. J. Heinzen, Phys. Rev. A46, R6797 (1992).

[2] A. Kuzmich, K. Mølmer and E. S. Polzik, Phys. Rev. 
Lett. 79, 4782 (1997).

[3] A. Kuzmich, N. P. Bigelow and L. Mandel, Europhys. Lett. 42, 481 (1998).

[4] E. S. Polzik, Phys. Rev. A59, 4202 (1999).

[5] J. Hald, J. L. Sørensen, C. Schori and E. S. Polzik, Phys. Rev. Lett. 83, 1319 (1999).

[6] A. Kuzmich, L. Mandel and N. P. Bigelow, Phys. Rev. Lett. 85, 1594 (2000).

[7] L. -M. Duan, J. I. Cirac, P.Zoller and E. S. Polzik, Phys. Rev. Lett. 85, 5643 (2000).

[8] A. Kozhenkin, K. Mølmer and E. S. Polzik, Phys. Rev. A62, 033809 (2000).

[9] A. Sørensen, L. -M. Duan, J. I. Cirac and P. Zoller, Nature (London) 409, 63 (2001).

[10] Z. Ficek and R. Tanaś, Phys. Rep. 372, 369 (2002).

[11] L. -M. Duan, E. Demler and M. D. Lukin, Phys. Rev. Lett. 91, 090402 (2003).

[12] B. Yurke, Phys. Rev. Lett. 56, 1515 (1986).

[13] M. Kitagawa and M. Ueda, Phys. Rev. Lett. 67, 1852 (1991).

[14] D. J. Wineland, J. J. Bollinger, W. M. Itano and D. J. Heinzen, Phys. Rev. A50, 67 (1994).

[15] M. A. Neilsen and I. L. Chaung, Quantum Computation and Quantum Information (Cambridge University Press, Cambridge, 2000).

[16] M. Kitagawa and M. Ueda, Phys. Rev. A47, 5138 (1993).

[17] G. S. Agarwal and R. R. Puri, Phys. Rev. A41, 3782 (1990).

[18] M. D. Lukin, S. F. Yelin and M. Fleishhauer, Phys. Rev. Lett. 84, 4232 (2000).

[19] X. Wang, A. Sørensen and K. Mølmer, Phys. Rev. 64, 053815 (2001); X. Wang, J. Opt. B: Quantum Semiclassical Opt. 3, 93 (2001); X. Wang, Opt. Commun. 200, 277 (2001).

[20] A. Sørensen and K. Mølmer, Phys. Rev. Lett. 86, 4431 (2001).

[21] C. Orzel, A. K. Tuchman, M. L. Fenselau, M. Yasuda and M. A. Kasevich, Science 291, 2386 (2001).
[22] A. Sørensen, Phys. Rev. A65, 043610 (2002).

[23] A. André and M. D. Lukin, Phys. Rev. A65, 053819 (2002).

[24] A. R. Usha Devi, K. S. Mallesh, M. A. A. Sbaih, K. B. Nalini and G. Ramachandran, J. Phys. A: Math. Gen 36, 5333 (2003).

[25] A. R. Usha Devi, X. Wang and B. C. Sanders, Quantum Inf. Proc. 2, 207 (2003).

[26] D. Ulam-Orgikh and M. Kitagawa, Phys. Rev. A64, 052106 (2001).

[27] X. Wang and B. C. Sanders, Phys. Rev. A68, 012101 (2003).

[28] J. K. Korbicz, J. I. Cirac and M. Lewenstein, Phys. Rev. Lett. 95, 120502 (2005).

[29] The qubit labels 1 and 2 in $\left\langle\left(\sigma_{1 i} \sigma_{2 j}\right)\right\rangle$ are only instructive. Infact, the average values of two qubit correlations are identical for any random pair of qubits belonging to a symmetric $N$-qubit system.

[30] N. Linden, S. Popescu and A. Sudbery, Phys. Rev. Lett. 83, 243 (1999).

[31] A. Sudbery, J. Phys. A: Math. Gen. 34, 643 2001).

[32] Y. Makhlin, Quantum Inf. Proc. 1, 243 (2003).

[33] S. Albeverio, Shao-Ming Fei, Preeti Parashar and Wen-Li Yang, Phys. Rev. A68, 010303(R) (2003).

[34] A symmetric state transforms into another symmetric state under identical local unitary transformation on both the qubits.

[35] R. Horodecki and M. Horodecki, Phys. Rev. A54, 1838 (1996).

[36] From the unit trace condition $t_{1}+t_{2}+t_{3}=1$ and the positivity condition $-1 \leq t_{1}, t_{2}, t_{3} \leq 1$, it is clear that $\operatorname{det} T<0$ also leads to $\operatorname{Tr}\left(T^{2}\right)>1$ i.e., $\mathcal{I}_{1}<0$ and $\mathcal{I}_{2}>1$ both imply pairwise entanglement.

[37] R. H. Dicke, Phys. Rev. 93, 99 (1954).

[38] C. A. Sackett et al., Nature 404, 256 (2000).

[39] D. M. Brink and G. R. Satchler, Angular Momentum (Oxford University Press, Oxford, 1975). 